\title{
Home-based fundamental approach to alleviate low back pain using myofascial release, stretching, and spinal musculature strengthening during the COVID-19 pandemic
}

\author{
Ardalan Shariat ${ }^{\mathrm{a}}$, Albert T. Anastasio ${ }^{\mathrm{b}}$, Sahar Soheilic and Mitch Rostad ${ }^{\mathrm{d}, *}$ \\ ${ }^{a}$ Sports Medicine Research Center, Neuroscience Institute, Tehran University of Medical Sciences, Tehran, Iran \\ ${ }^{\mathrm{b}}$ Department of Orthopaedic Surgery, Duke University, Durham, NC, USA \\ ${ }^{\mathrm{c}}$ Department of Biomechanics and Sport Injury, Faculty of Physical Education and Sports Sciences, Kharazmi \\ University, Tehran, Iran \\ d Organ Preservation Alliance, Berkeley, CA, USA
}

Received 14 July 2020

Accepted 28 July 2020

\begin{abstract}
. (LBP) is likely to rise as sedentary behavior increases. associated LBP exacerbated during the COVID-19 pandemic. evidence-based pain reduction strategies. decreased and, in some cases, cured.

\footnotetext{
*Address for correspondence: Dr. Mitch Rostad (M.D.), Organ
} Preservation Alliance, 950 Gilman Street, STE 200, Berkeley, California, 94710, USA. Tel.: +1 612250 9969; E-mail: mitch.rostad@organpreservationalliance.org.
\end{abstract}

BACKGROUND: The COVID-19 pandemic has caused global adoption of stay-at-home mandates in an effort to curb viral spread. This lockdown has had the unintended consequence of decreasing physical activity, and incidence of low back pain

OBJECTIVE: In this article, we aim to provide a fundamental, novel approach to alleviate LBP including desk worker

METHODS: Individuals can alleviate their LBP through a simplistic, self-therapeutic approach: myofascial release and stretching of key musculature involved in LBP following a simple technique and associated time domain, as well as a 360degree strengthening of the muscles surrounding the lower back. Additional muscular strength will support the lower back and lend resilience to aid in the mitigation of pain caused by poor work-related postural positions.

RESULTS: We demonstrate several exercises and movements aimed at alleviating LBP. Additionally, we provide a summary graphic which facilitates ease of use of the exercise plan and represents a novel methodology for simple distribution of

CONCLUSIOS: Through mitigation of sedentary behavior and adoption of the techniques described herein, LBP can be

Keywords: Functional strength, fitness, self-therapeutic, resilience, posture, COVID-19

\section{Introduction}

Lower back pain (LBP) is a significant contributor to morbidity and lack of functionality worldwide [1]. Globally, the prevalence of activity-limiting has 
been demonstrated to be roughly $12 \%$ for LBP lasting greater than one day and for those with LBP, as high as $25 \%$ of patients will have pain lasting for greater than one month [2]. Given the current COVID-19 global pandemic, wide-spread shelter-in-place has led to an overall decrease in activity [3]. With a substantially increased risk of onset of LBP with increased daily sitting duration $[4,5]$, it is reasonable to assume the prevalence of LBP may continue to rise as the COVID-19 quarantine continues [6]. Surgical treatment of LBP is a maximally invasive intervention and should be reserved for refractory cases [7, 8]. As an alleviating factor for LBP, regular physical exercise is known to treat pain symptoms for this condition and is an important part of maintaining comprehensive health [9-11]. This article provides a fundamental, minimalistic approach to alleviate and protect against LBP by targeting primary muscles involved including the quadratus lumborum, gluteus muscle complex, and iliopsoas utilizing the techniques of myofascial release, stretching, and spinal stability strengthening. This routine is a feasible option for individuals quarantined at home who are unable to attend a gymnasium or physical therapy facility. Additionally, these techniques are low cost and do not require extensive equipment for their completion.

\section{Myofascial release}

Myofascial release, also colloquially known as "soft tissue smash" involves the process of using a tool, typically a lacrosse ball or soft yoga smash ball, to decrease tension in tight musculature by applying pressure. This method of personal therapy is useful to alleviate muscular tension at joints and therefore alleviate pain $[12,13]$. Myofascial release can be done in a low cost manner and is very easy to perform after a simple coaching or instructional session.

While many of the following exercises have previously been identified and validated in the evidence-based literature, we present a novel, simple approach for combining them to aid in the reduction and prevention of LBP. Furthermore, several methodologies are presented in this article for better targeting specific muscle groups for myofascial release.

The following figures will show how to: 1 . survey the musculature for tightness, 2 . alleviate tension by constant pressure, 3. alleviate tension by incorporating movement ("pressure wave" technique, moving muscle through range of motion). Surveying the musculature involves lightly tracing the musculature from origin to insertion. Once a tight spot has been identified, spend a total of two minutes minimum on that tight spot. Evidence suggests that a minimum dose of 90 seconds is appropriate for soft tissue change [14] and to keep our method consistent with recommended doses of stretching exercises, we believe in a two-minute approach. Exercises can be repeated throughout the day, with careful attention to the "red flag" signs (severe pain, numbness, tingling). Additionally, we provide a graphic summary (Fig. 13) which allows for ease in memorization of the routine. This novel approach to distribution of evidence-based exercises is designed to facilitate consistency in completion of the movements.

For the first minute, breath, relax, and use your own body weight to apply pressure to the tight area. Then, for the second minute, consider incorporating movement by either taking the muscle through the full range of motion or by applying a "pressure wave" or a slight gyration of the lacrosse ball. As the body adapts and muscle begin to loosen, more intensity can be added by holding weights while applying pressure on the lacrosse ball. See photographs below for specifics on high priority muscles that cause LBP.

\subsection{Quadratus lumborum ( $Q L)$}

Find the muscle by creating a box as seen in Fig. 1. Place the ball in the center of the box and lie down onto your back (Fig. 2). To ensure that the ball is in the proper place, abduct your knee slowly to the ground, taking the QL through its range of motion. Once confirmed, follow the process outlined above.

\subsection{Gluteus muscular complex}

Find the muscles by using your greater trochanter and gluteal cleft as landmarks (Fig. 3). Place the ball on your glutes and lie down onto your back (Fig. 4). Once you are in position, follow the process outlined above. If you suffer from LBP, it is recommended that you spend extra time on your gluteus medius. Range of motion will involve abduction of the knee to the ground.

\subsection{Iliopsoas}

Find the muscles by locating your anterior superior iliac spines (ASIS) indicated by the right index finger in Fig. 5. Place the ball immediately on the 

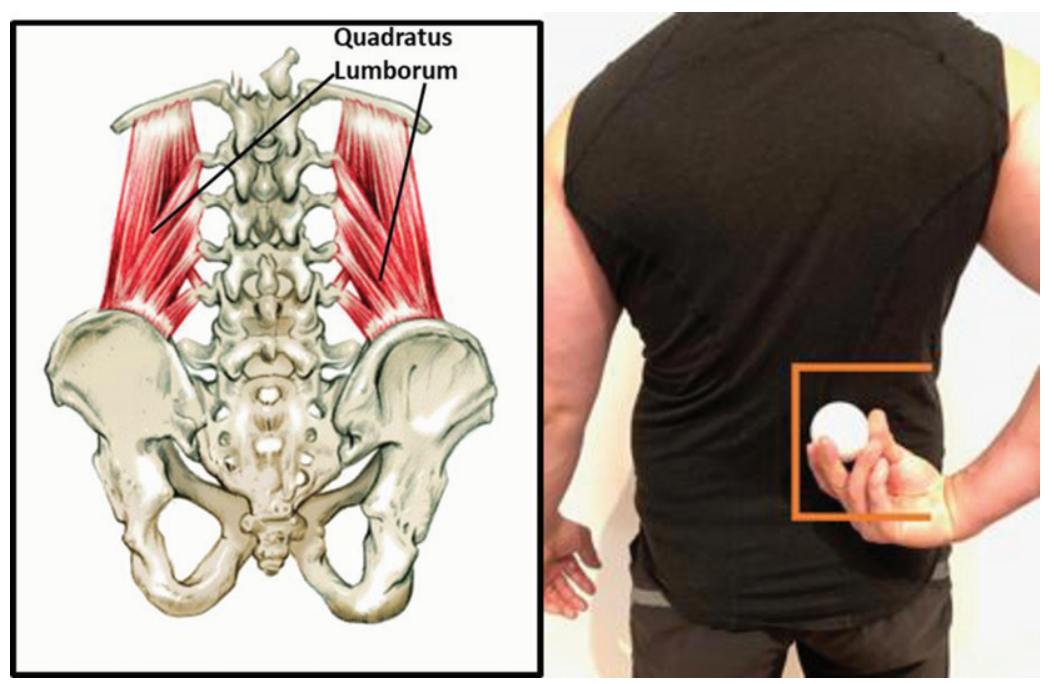

Fig. 1. Find the muscle by creating a box as seen in the photo: bottom ribs, spine, top of glutes.

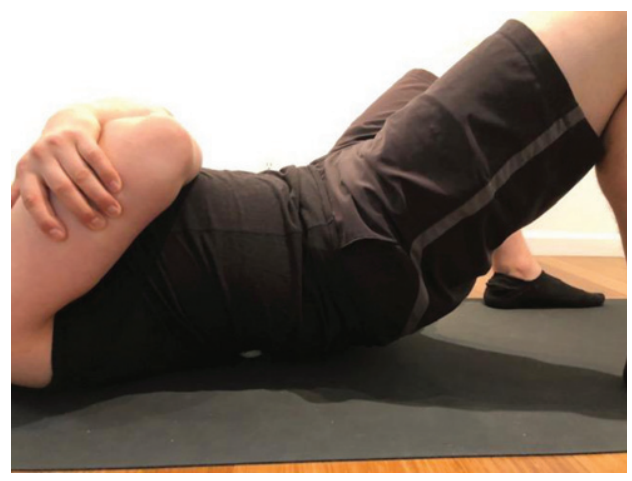

Fig. 2. Place the ball in the center of the box and lie down onto your back. medial side of the ASIS. Here you will have located both your iliacus and psoas (major) muscles. Spend two minutes in this location. Sometimes feeling an appropriate pressure on this spot can be difficult. Therefore, we recommend elevating the ball with either a book or a yoga block. Additionally, you can externally rotate your leg as the same side as the ball to bring the muscles closer to the surface. For an additional two minutes, move the ball into position two, which focuses solely on your psoas (major and minor). You obtain this position by moving the ball diagonally from position one towards your umbilicus. Stop before you reach your umbilicus. Spend two minutes at this spot (Fig. 6).
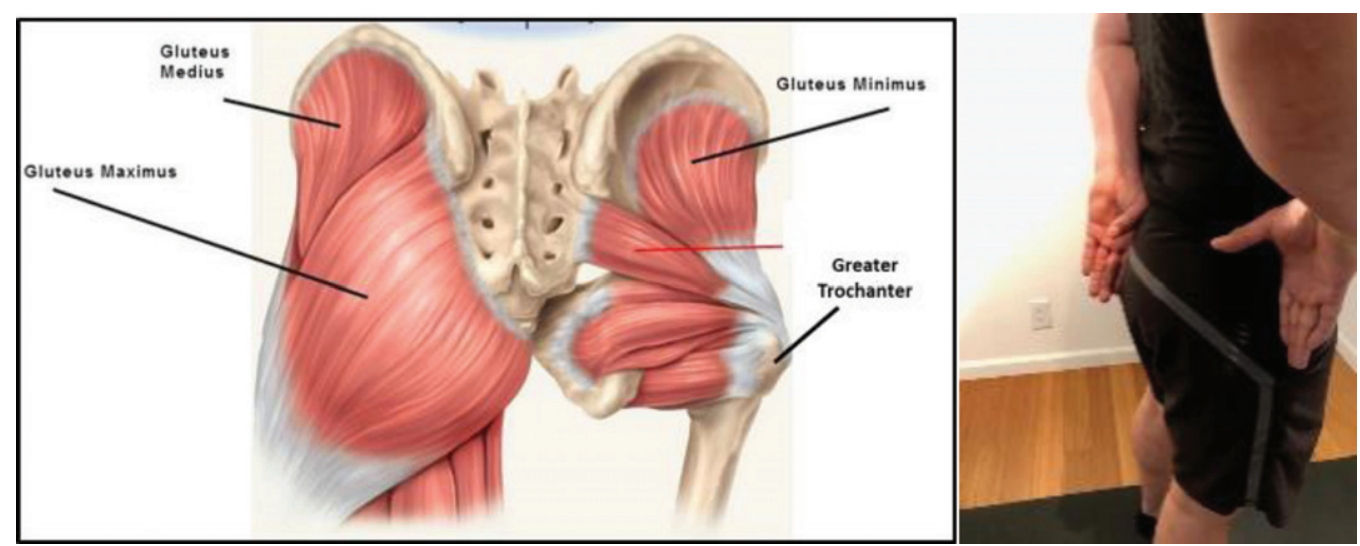

Fig. 3. Find the muscles by using your greater trochanter and gluteal cleft as landmarks. 


\section{Stretching techniques}

Even though a number of sets, timed repetitions, and techniques exist for stretching, continuing the simple approach of two minutes per stretch has proven to be most effective [15]. You can perform

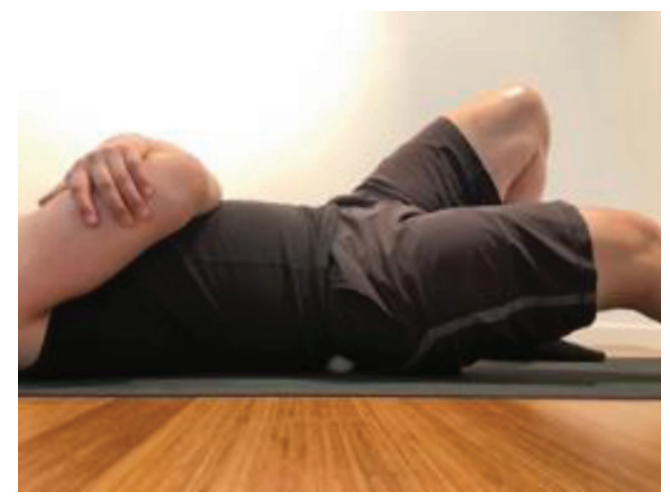

Fig. 4. Place ball on your glutes and lie down onto your back. these stretches as a static hold or, after a minute of static hold, incorporate movement by lightly moving in and out of the stretch position that provides light tissue restriction.

\subsection{QL $90-90$ stretch}

For this stretch, choose a hip to place behind you and form a 90-degree angle at the knee. Place the other foot at a 90-degree angle in front of you. As you reach forward with your left hand, push your left hip away from the outstretched hand. Try to elongate the area of the quadratus lumborum. You can also try leaning forward to intensify the stretch (Fig. 7).

\subsection{Glutes stretch}

For this stretch, lie on your back with knees bent and cross the leg of the glutes that you want to stretch across the knee of the other leg. Hold onto the hamstrings on the non-crossed over leg, lacing the fingers.
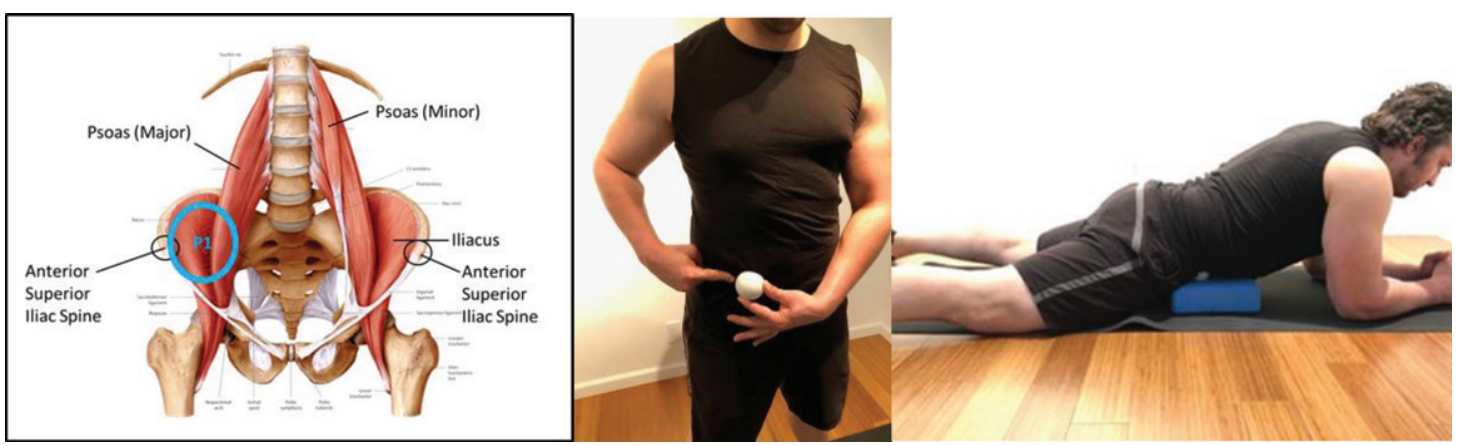

Fig. 5. Find the muscles by locating your anterior superior iliac spines (ASIS) indicated by the right index finger (left). Place the ball immediately on the medial side of the ASIS (right).
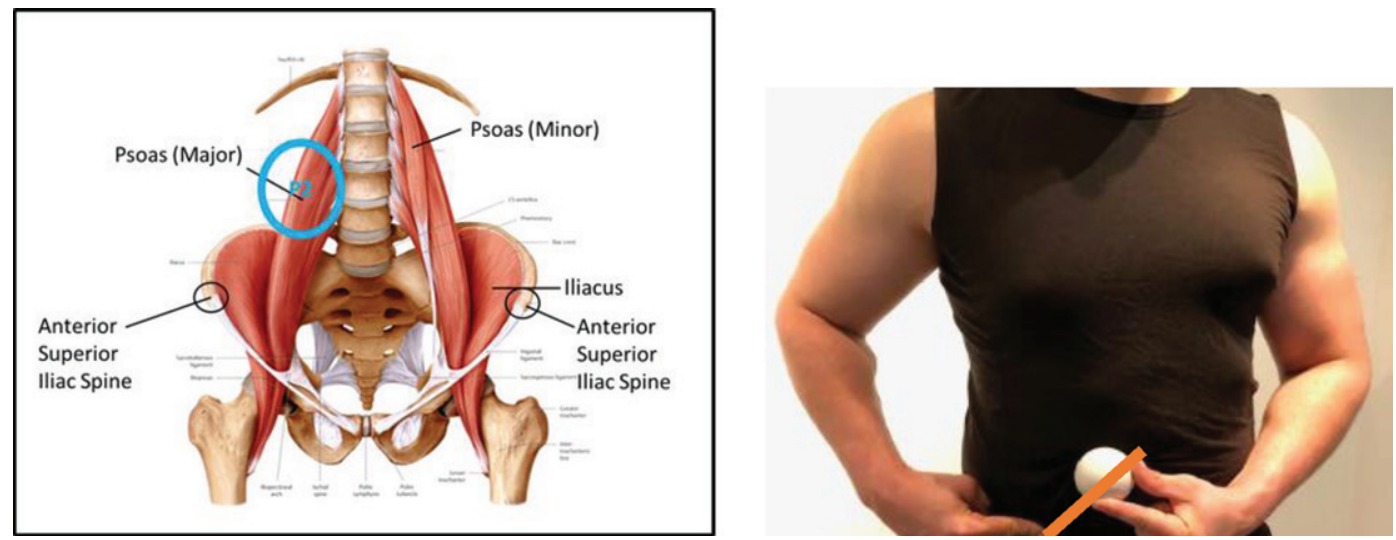

Fig. 6. Spend two minutes at the psoas to target the major and minor muscles. 
Lightly pull back and feel the stretch. Follow the process outlined above (Fig. 8).

\subsection{Iliopsoas/hip flexors free standing stretch}

Position yourself into a lunge position. You may want to place some padding underneath your knee. Make sure that you brace your core and glutes so that your pelvis remains aligned throughout the stretch and does not bias to either anterior or posterior pelvic tilt. Now lean forward and feel a stretch in your hip flexors. You can intensify the stretch by place your hands behind your head, tilting away from the side where you knee is down and rotating away from that same side with your arms (Fig. 9).

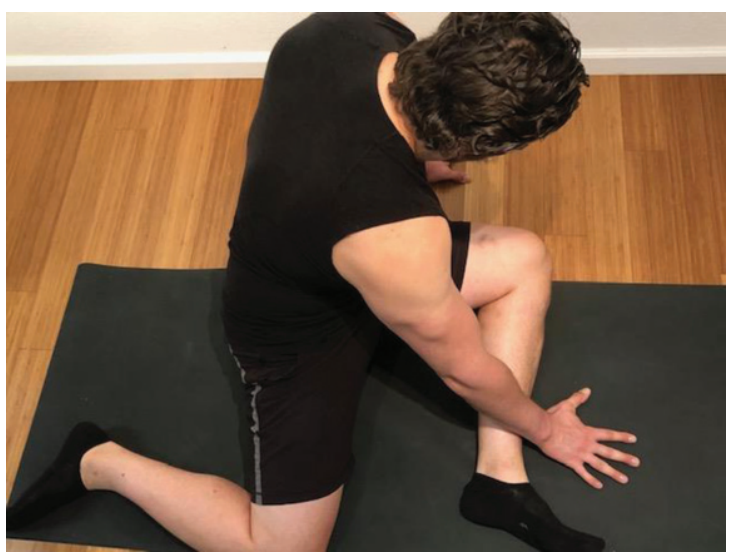

Fig. 7. QL $90-90$ stretch.

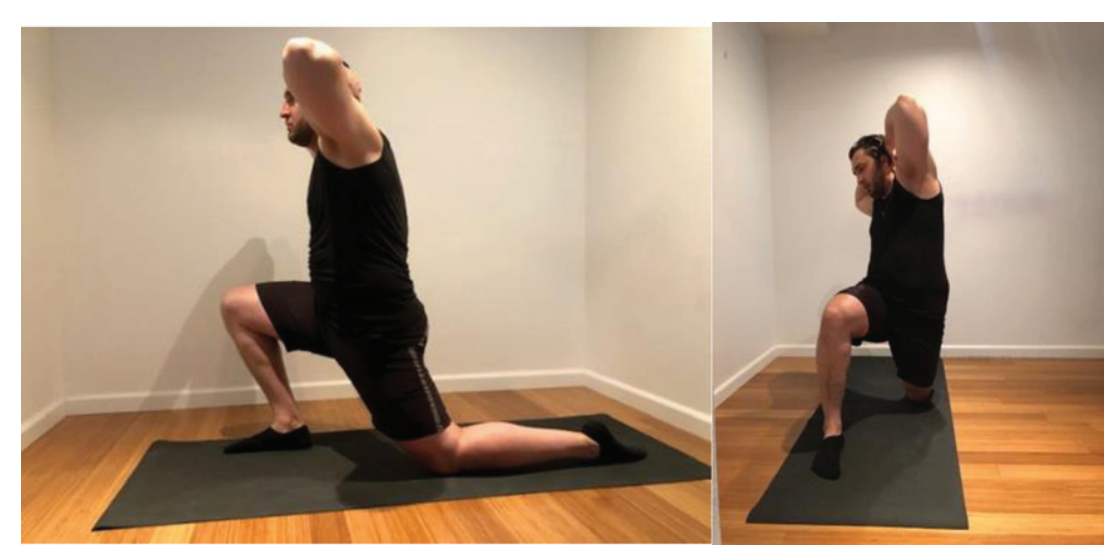

Fig. 9. Iliopsoas/hip flexors free standing stretch.

\subsection{Cat-camel}

\section{McGill Big 3: Spine stabilization and strengthening}

The McGill Big 3 exercises are aimed at strengthening spinal stability and resilience. Dr. Stuart McGill has investigated the causal mechanisms of lower back pain and has published work aimed at rehabilitating those suffering from back pain with additional emphasis on improving resilience and performance $[16,17]$.

Prior to the start of this workout, it is recommended to do a set of warm up exercises, such as the cat-camel movement. In addition to warming up the body for the activity, this exercise also improves the movement of the spine and reduces stiffness of the lower back muscles.

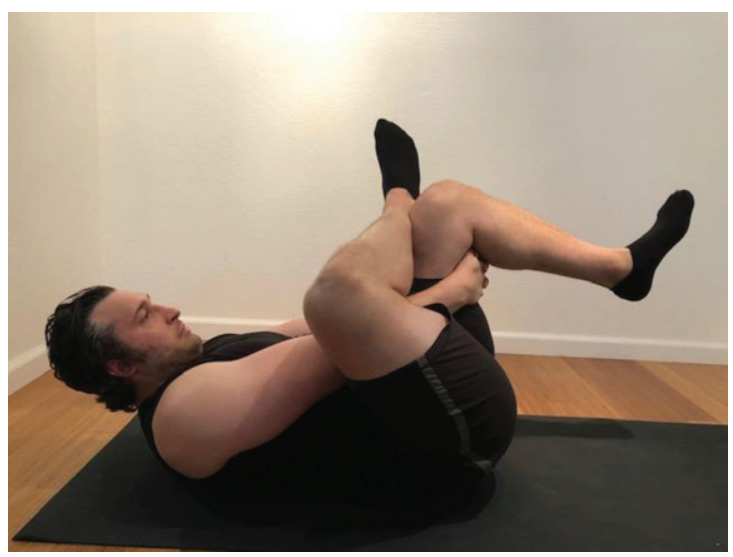

Fig. 8. Glutes stretch. 


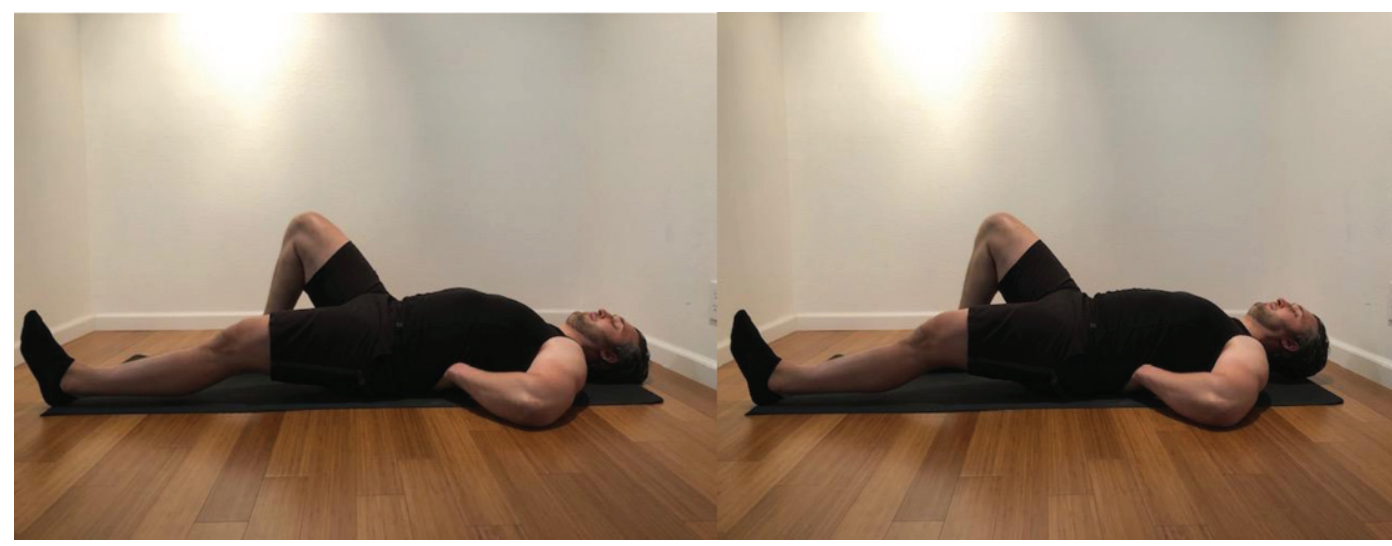

Fig. 10. The curl up.

Step 1: Get on your hands and knees (as if you were a quadrupedal animal).

Step 2: First, slowly begin to raise your spine and hips to create a curved or rounded back (the camel). End this movement by lowering your head to the ground while your neck is in line with your chin.

Step 3: Pause after a few seconds, while the head is looking up, try to move in the opposite downward extended position (the cat).

Perform this movement 5-6 times before starting the McGill Big 3 exercises.

\subsection{The curl up}

Step 1: Lie on your back with one knee bent and the other straight. If you feel pain in one of your legs, this leg can remain flat. Next, place your hands under your back.

Step 2: Lift your head just a few inches off the ground and hold for 10 seconds. During the rest period, imagine your head to be on a pillow, utilizing this imagery to raise the head. It is imperative that during the curl up, there be no movement in the lower back. Additionally, if a participant lifts their head and shoulders too high, it will put a lot of pressure on the spine, which can increase pain symptoms.

Step 3: Hold for 10 seconds, then bring down the head to a resting position (Fig 10). We recommend performing this movement with a minimum of three sets with 10 seconds rest between each set. Hold the position for additional time as stamina allows.

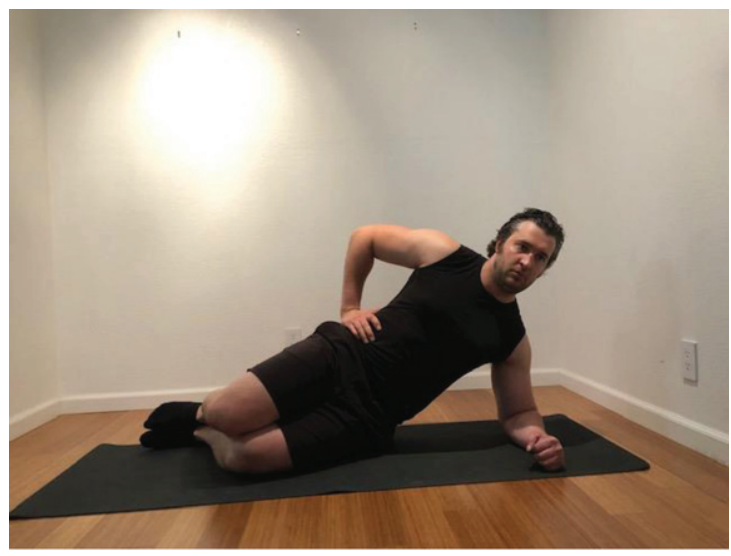

a: the side plank

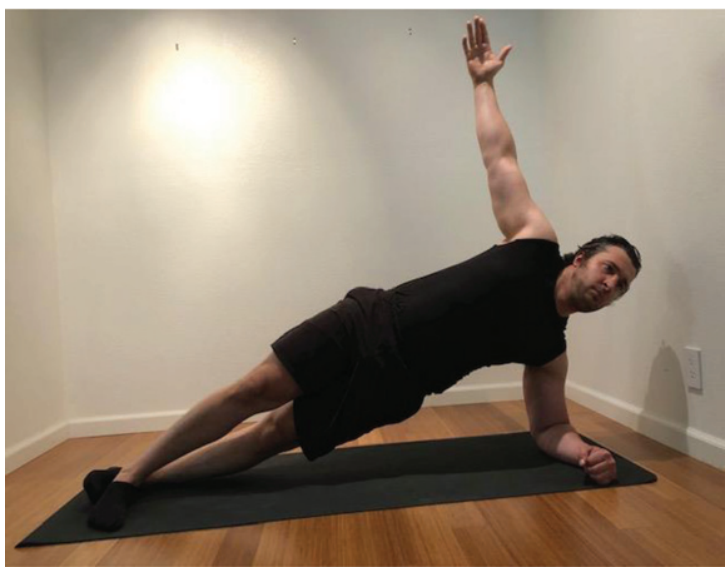

b: a straight leg plank

Fig. 11. a) The side plank. b) A straight leg plank. 


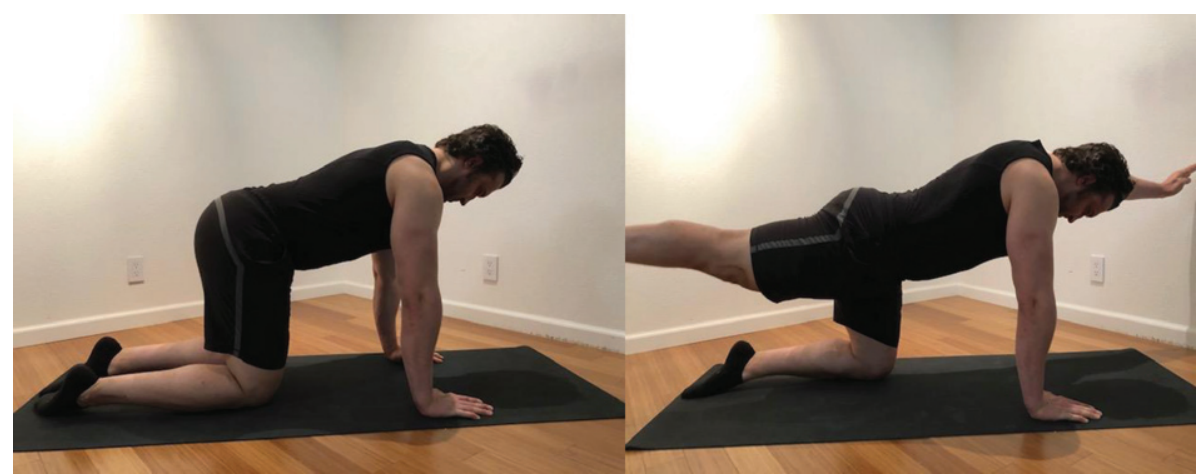

Fig. 12. The bird dog.

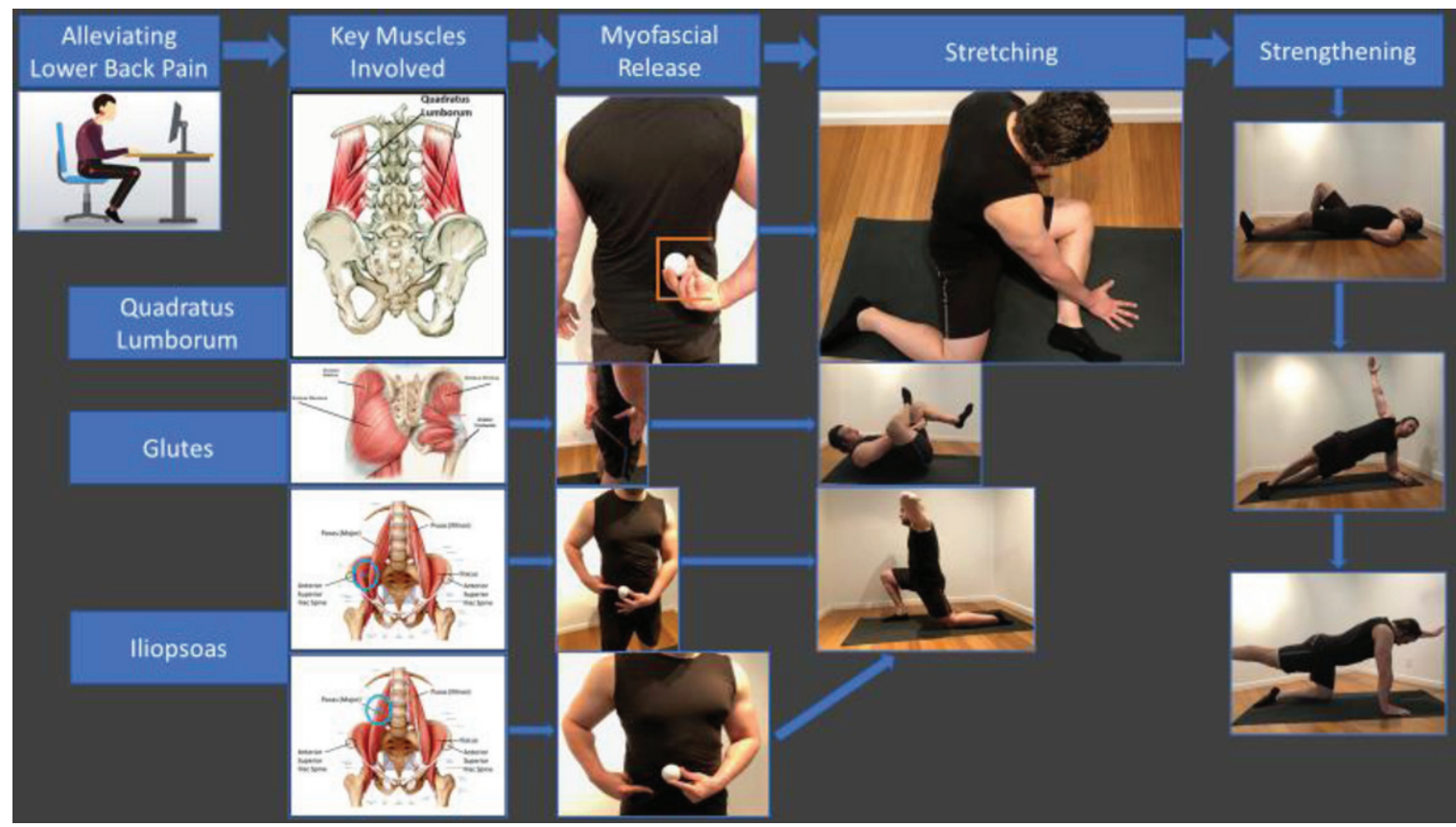

Fig. 13. Summary of the movements aimed at alleviating low back pain exacerbated by ecreased physical activity brought on by the lockdown during the COVID-19 pandemic.

\subsection{The side plank}

Through side plank training, by activating the lateral oblique muscles and QL, an individual can retrain their spine to improve alignment. Thus, it is a particularly effective exercise to help with weaknesses in stability.

Step 1: With your legs bent at the knees, lie on one side of your body while supporting your upper body with your elbows, placing your free hand on the opposite shoulder.
Step 2: Once your knee and arm support your body, raise the hip directly towards the ceiling of the room.

Step 3: Hold this position for 10 seconds before returning the hip back to the floor. Repeat the exercise on each side. We recommend performing this movement with a minimum of three sets with 10 seconds rest between each set. Hold the position for additional time as stamina allows. For an advanced option, Fig. 11 shows two straight leg options for those desiring additional difficulty. 


\subsection{The bird dog}

The McGill Big 3 workout ends with the completion of the bird dog movement. This is a particularly effective exercise to maintain tension in the core muscles. Core stability has an important effect on spinal alignment and aids in the completion of normal dayto-day activities.

Step 1: Assume the fourth position (quadruped) with the back in a neutral stance. Remember that in the neutral position, a very slight curve is desired in the lower back, rather than a straight back stance.

Step 2: Without any movement in the low back, pull one leg back while lifting the opposite arm at the same time until both limbs are completely straight. A way to help ensure that foot movement is not causing an overarching of the back is to envision a kick back with the heel.

Step 3: Hold this position for 10 seconds before returning to the all four position. Do not allow the back to bend during the movement. Follow the same exercise layout as in the previous two exercises. We recommend performing this movement with a minimum of three sets with $10 \mathrm{sec}-$ onds rest between each set. Hold the position for additional time as stamina allows.

\section{Conclusion}

The incidence of lower back pain (LBP) is likely to continue to increase globally as the COVID-19 pandemic lockdown causes perturbations in the daily activity schedule of the general public. Thus, a simple, yet functional-based approach which can be undertaken at home with minimal equipment may prove both therapeutic and preventative. Herein, we propose a novel approach to alleviate LBP through the use of various myofascial release, stretching, and strengthening techniques.

\section{Acknowledgments}

The authors wish to thank the Ayushi Agrawal for taking the photographs and Dr. Kelly Starrett for foundational mobility inspiration.

\section{Conflict of interest}

No potential conflict of interest relevant to this article was reported.

\section{References}

[1] Shariat A, Cardoso JR, Cleland JA, Danaee M, Ansari NN, Kargarfard M, Mohd Tamrin SB. Prevalence rate of neck, shoulder and lower back pain in association with age, body mass index and gender among Malaysian office workers. Work. 2018;60(2).

[2] Hoy D, Bain C, Williams G, March L, Brooks P, Blyth F, Woolf A, Vos T, Buchbinder R. A systematic review of the global prevalence of low back pain. Arthritis Rheum 2012;64(6):2028-37.

[3] Woods J, Hutchinson NT, Powers SK, Roberts WO, GomezCabrera MC, Radak Z, Berkes I, Boros A, Boldogh I, Leeuwenburgh C. The COVID-19 Pandemic and Physical Activity. Elsevier; 2020.

[4] Gupta N, Christiansen CS, Hallman DM, Korshøj M, Carneiro IG, Holtermann A. Is objectively measured sitting time associated with low back pain? A crosssectional investigation in the NOMAD study. PLoS One. 2015;10(3):e0121159.

[5] Shariat A, Tamrin SBM, Arumugam M, Danaee M, Ramasamy R. Comparative reliability of different instruments used to measure the severity of musculoskeletal disorders in office workers. Work. 2016;54(3).

[6] Shariat A, Hakakzadeh A, Cleland J. Home-based exercise note in Covid-19 quarantine situation for office workers: A commentary. Work. 2020;(Preprint):1-2.

[7] Anastasio AT, Niu S, Kim EJ, Rhee JM. Evaluating Single-Surgeon Bias Toward Recommending Corrective Procedures for Cervical Spondylotic Myelopathy Based on Demographic Factors and Comorbidities in a 484-Patient Cohort. Glob Spin J. 2020;2192568219896296.

[8] Niu S, Anastasio AT, Faraj RR, Rhee JM. Evaluation of Heterotopic Ossification After Using Recombinant Human Bone Morphogenetic Protein-2 in Transforaminal Lumbar Interbody Fusion: A Computed Tomography Review of 996 Disc Levels. Glob Spin J. 2020;10(3):280-5.

[9] Wang R, Weng L-M, Peng M-S, Wang X-Q. Exercise for Low Back Pain: A Bibliometric Analysis of Global Research from 1980 to 2018. J Rehabil Med. 2020;52(4): $1-11$.

[10] Alizadeh R, Shariat A, Hakakzadeh A, Selk-Ghaffari M, Damavandi P, Honarpishe R, Ingle L. A comprehensive screening protocol to identify incidence of lower back pain in military office workers. J pain manage. 2020.

[11] Shariat A, Alizadeh R, Moradi V, Afsharnia E, Hakakzadeh A, Ansari NN, Ingle L, Shaw BS, Shaw I. The impact of modified exercise and relaxation therapy on chronic lower back pain in office workers: a randomized clinical trial. J exer rehab. 2019;15(5):703.

[12] Beardsley C, Škarabot J. Effects of self-myofascial release: a systematic review. J Bodyw Mov Ther. 2015;19(4): $747-58$

[13] Kim Y, Hong Y, Park H-S. A soft massage tool is advantageous for compressing deep soft tissue with low muscle tension: Therapeutic evidence for self-myofascial release. Complement Ther Med. 2019;43:312-8. 
[14] Hughes GA, Ramer LM. Duration of myofascial rolling for optimal recovery, range of motion, and performance: a systematic review of the literature. Int J Sports Phys Ther. 2019;14(6):845.

[15] Page P. Current concepts in muscle stretching for exercise and rehabilitation. Int J Sports Phys Ther. 2012;7(1):109.
[16] Cannon J, Emond D, McGill SM. Evidence on the Ability of a Pneumatic Decompression Belt to Restore Spinal Height Following an Acute Bout of Exercise. J Manipulative Physiol Ther. 2016;39(4):304-10.

[17] Lee B, McGill S. The effect of short-term isometric training on core/torso stiffness. J Sports Sc. 2017;35(17):1724-33. 